\title{
Pneumococcal vaccination in celiac disease
}

\author{
Giovanni Casella ${ }^{a}$, Fabio Ingravalle ${ }^{b}$, Giorgio Abbate ${ }^{c}$, Claudio Monti ${ }^{a}$, Fulvio Bonetti ${ }^{a}$, Gabrio Bassotti ${ }^{d}$ \\ Pasquale Mansueto ${ }^{\mathrm{e}}$, Vincenzo Villanaccif and Antonio Carroccio ${ }^{\mathrm{e}, \mathrm{g}}$
}

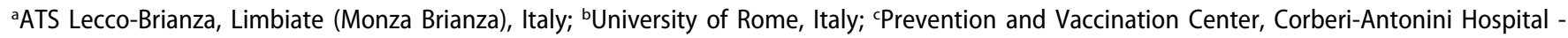
ASST Monza, Limbiate (Monza Brianza), Italy; 'Gastroenterology, Hepatology and Digestive Endoscopy Section, Department of Medicine, University

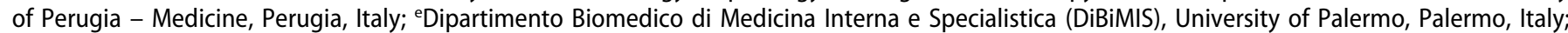
fPathology Department, Spedali Civili Brescia, Brescia, Italy; Internal Medicine, Giovanni Paolo II Hospital, Sciacca (ASP Agrigento), Italy

\section{ABSTRACT}

Introduction: Celiac disease $(C D)$ is an immune-mediated disorder associated with gluten exposure in genetically predisposed subjects.

Areas covered: Infectious disease is one of the causes of morbidity and mortality in CD patients. Invasive streptococcus pneumoniae (pneumococcus) is a particularly dangerous morbid condition in both the general population and celiac patients. Pneumococcal vaccination is the most effective means for its prevention.

Expert opinion: In CD, evaluation of spleen function should be useful to select patients who may benefit from vaccination to reduce the risk of pneumococcal disease. Different strategies could be employed: physicians could search for signs of hyposplenism on peripheral blood smear or abdominal ultrasound. However, the best strategy to identify which patients will benefit from pneumococcal vaccination has not yet been defined.
ARTICLE HISTORY

Received 16 January 2019

Accepted 10 April 2019

KEYWORDS

Celiac disease;

hyposplenism; infections; pneumococcal vaccination; pneumonia

\section{Introduction}

Celiac disease (CD) is a chronic immune-mediated gastrointestinal disorder found in more than $1 \%$ of the Western population and caused by gluten exposure in genetically predisposed subjects. It is strongly associated with HLA DQ-2 and DQ-8 haplotypes, and exposure to diets containing gluten can lead to the development of the disease with its typical and atypical symptoms, associated with the development of circulating autoantibodies (anti-transglutaminase and anti-endomysial antibodies), and duodenal histology alterations, ranging from mild (increased intraepithelial lymphocytes) to severe (villous atrophy). A glutenfree diet is the only efficacious treatment for $C D[1,2]$.

In addition to its several clinical manifestations, CD is also associated with a predisposition for infectious disease, which can increase morbidity and mortality. Several studies have reported an increased risk of urinary tract infections [3], tuberculosis infection [4], invasive pneumococcus infection [5,6], and sepsis [7].

Many factors can influence immune response in $C D$ patients. A decrease in splenic function due to

hyposplenism or atrophy is often detected in $C D$, possibly resulting in a reduced response to bacterial infections [8].

The present paper aims to review the causes of the increased risk of infection in $C D$ and the evidence regarding pneumococcal infection and vaccination in CD.

\section{Hyposplenism and infections in CD}

The importance of the spleen in maintaining the immunoglobulin (Ig)M memory B-cell pool has been demonstrated, as well as its fundamental role in protecting against infection by encapsulated bacteria. Memory B cells, produced in response to infectious agents or vaccines, persist in the organism and rapidly produce antibodies following a second stimulation with the same pathogen. In humans, about half of $B$ cells express CD27 and are considered memory $B$ cells. IgM memory $B$ cells have different origins and functions from switched memory $B$ cells. They are not present in splenectomized and asplenic patients, whereas switched memory B cells are only transiently depleted after splenectomy and are normally detectable in asplenic patients. IgM memory $B$ cells originate from the spleen marginal zone, which supports lgM memory B cell survival. These cells produce natural antibodies (mostly of IgM type) necessary for T-independent response to encapsulated bacteria. By opsonizing invading pathogens, natural antibodies also play a crucial role in activating the complement cascade and amplifying early innate defense mechanisms [9]. More specifically, an impairment of the immune function of the spleen results in: (a) a reduction in IgM-memory $B$ cells and a defective opsonization activity, thus predisposing to infections caused by encapsulated bacteria (mainly Streptococcus pneumoniae, Neisseria meningitidis and Haemophylus influenzae); and (b) a decrease in marginal zone B cells which, in turn, predisposes to the emergence of autoreactive T-cell clones, as a consequence of contemporary T-regulatory cell depletion, with the subsequent development of autoimmunity. Moreover, it must be remembered that the spleen also has an important filtering function; impairment results in (a) the defective removal of pits from erythrocytes, with a consequent increase in circulating pitted red cells and Howell-Jolly bodies, and (b) reduced platelet 
Article highlights

- Impairment of the immune function of the spleen results in a reduced number of IgM-memory B cells, the emergence of autoreactive T-cell clones, defective removal of pits from erythrocytes, and reduced platelet sequestration, with thrombocytosis

- Hyposplenism, a well-known risk factor for pneumococcal infection, is not routinely investigated and evaluated in celiac patients

- Every physician should investigate blood and/or ultrasound signs of hyposplenism and splenic atrophy in CD patients

- A systematic evaluation of spleen function should permit the selection of CD patients who may benefit from vaccination, in order to reduce the risk of invasive pneumococcal disease

- Celiac patients with impaired immunologic function need to undergo a complete vaccination protocol, including $S$. pneumoniae, $N$. meningitidis, $H$. influenzae type $B$, and influenza.

sequestration, leading to thrombocytosis, which, in turn, predisposes to thromboembolism $[10,11]$.

Consequently, in splenectomized and asplenic patients, a contemporaraneous impairment of both the immune and filtering function has been shown, and a significant inverse correlation between levels of circulating IgM memory B cells (detected by flow-cytometry) and pitted red cell values has been reported in inflammatory bowel disease patients with hyposplenism [12].

Therefore, in accordance with Di Sabatino et al. [11], we can suggest that $C D$ patients should be considered as suffering from major hyposplenism when an lgM memory B cell frequency lower than 10 and/or a pitted red cell value higher than $4 \%$ are present, indicating that this population could receive more clinical benefits from pneumococcal vaccination.

Pitted red cell count can be considered as the reference method for diagnosing hyposplenism. Although this approach could be considered simple, 'pit identification' (a pit being the characteristic depression on the erythrocyte surface) in a blood smear requires the use of special equipment (Zeiss/Nomarski differential interference contrast microscopy) which is not so readily available.

In addition to the presence of low IgM memory B cells, increased pitted red blood cells, and Howell-Joly bodies, simple evaluation of spleen size and volume by abdominal ultrasound can be considered the first step in detecting hyposplenism. In this context, Di Sabatino et al. showed that two-thirds of individuals with a small spleen (longitudinal diameter $<7.5-8 \mathrm{~cm}$, incidentally detected on abdominal ultrasound) had splenic hypofunction, with pitted erythrocyte levels comparable to those of splenectomized patients [13].

$C D$ is the most frequent of the various diseases associated with hyposplenism, which affects more than a third of celiac patients. It does not complicate $C D$ in infancy, whereas in adults its incidence correlates with the duration of previous exposure to gluten, and a gluten-free diet is effective in restoring splenic function. The prevalence of hyposplenism increases from $19 \%$ to $80 \%$ in celiac patients with autoimmune disorders (AID) and/or premalignant or malignant complications. Therefore, splenic atrophy is recognized as a factor associated with a worse prognosis in CD [14,15].

In this context, Di Sabatino et al. demonstrated that in patients with complicated CD and in celiac patients with AID the median percentage of pitted red cells $(9.3 \%$ and $4.9 \%$, respectively) was significantly higher than in uncomplicated celiac patients without AID (median 2.6\%; $P<.005$ ), and in nonceliac patients with AID (median pitted red cells $1.8 \% ; P<.001$ ) and healthy volunteers (median pitted red cells $0.9 \% ; P<.0001$ ). As expected, splenectomized patients had a significantly higher number of pitted red cells (median 15.8\%, range 8.4-2.5; $P<.005$ ) than patients with complicated $C D$. The prevalence of splenic hypofunction (pitted red cells $>4 \%$ ) was significantly higher in patients with complicated and AID-associated CD than in patients with uncomplicated CD without AID [15].

As quoted above, hyposplenism in $C D$ can cause severe infections, mainly due to encapsulated bacteria, such as Pneumococcus. This kind of infection triggers the response of natural IgM antibodies, produced by memory B-cell populations residing in the marginal zone of the spleen, which in turn are responsible for the activation of T-independent immune responses against encapsulated bacteria [11].

In CD patients, malnutrition and increased intestinal permeability may be other factors predisposing to an impairment of the immune system's ability to respond to infections [16]. Reduced serum levels of folate, vitamin B12 and vitamin $D$ are frequent in $C D$ patients and may contribute to the reduction in immunologic functions [17].

Increased intestinal permeability has been recognized as one of the events predisposing to the development of multiple organ failure in critically ill ICU patients [18], although this aspect has not yet been confirmed in CD patients. It is important to underline that, in addition to increased intestinal permeability, CD patients also show an increased permeability of the respiratory mucosa [19].

Streptococcus pneumoniae is a particularly significant infection in $C D$ patients; as a consequence, the British Society of Gastroenterology has recommended the vaccination of all CD patients against pneumococcal disease (grade C recommendation) [20].

The British Guidelines based this recommendation on the evidence that $C D$ is often associated with hypoplasia and that splenic atrophy is a known risk factor for encapsulated bacterial infections, such as Pneumococcus [21].

However, neither the American College of Gastroenterology nor other Gastroenterology societies have specifically recommended vaccination for celiac patients [22], whereas it is recommended, in general, for patients with splenic atrophy or asplenia [22].

\section{International literature data about the dimension of the problem}

Early evidence on the risk of streptococcus pneumoniae (Pneumococcus) infection and fatal septicemia in CD patients was provided only by case reports, which documented a lack of response to standard therapy and the absence of other risk factors than CD and hyposplenism [23-25].

It is currently being debated how frequently pneumococcal infections occur in $C D$ populations compared to the general population. Several studies have investigated this aspect, both before and after the current British Guidelines were introduced. 
Thomas et al. [5] reported that CD patients have a higher risk of invasive pneumococcal disease than the general population, with a rate ratio of 2.06 (95\% confidence interval, $\mathrm{Cl}$ 1.27-3.15) in an Oxford population and a rate ratio of 1.61 (95\% Cl 1.36-1.90) in England as a whole. A higher risk of pneumococcal infection in splenectomized patients was reported, with a rate ratio of $3.40(95 \% \mathrm{Cl} 2.44-4.60)$ in an Oxford population and 3.32 (95\% Cl 2.80-3.90) in England as a whole. The authors stressed that CD patients aged between 15 and 64 and over 65 had a higher risk than controls, whereas in patients under 15 years of age there was no significant difference in risk of pneumococcal infections.

Rochert Tjemberg et al. [6], in a population-based cohort study on invasive pneumococcal disease in Sweden, showed an increased risk in $C D$ patients about $46 \%$ higher than in the general population. The study included 29,012 individuals with biopsy-proven CD, matched with 144,257 controls, and the infection events of Pneumococcus were identified through regional and national microbiological databases. Recorded pneumococcal infection was $45 / 29,012$ in the CD patients (0.15\%) and 162/ 144,257 in controls (0.11\%) (Hazard Risk, HR 1.46, 95\% Cl 1.05-2.03). The authors suggested that CD subjects and their physicians should consider pneumococcal vaccination.

Similarly, Ludvigsson et al. [7] evaluated the risk of sepsis in CD subjects. These authors used the Swedish national health registers to identify 15,325 subjects with a diagnosis of celiac disease and 14,494 inpatient reference individuals. The risk of sepsis was higher than in the control populations, with an HR of 1.6 (95\% Cl 1.2-1.9), versus the inpatient reference individuals, and an HR of $2.6(95 \% \mathrm{Cl} 2.1-3.0)$, versus the general population, respectively. A strong association was found with sepsis due to pneumococcal infection, with an HR of 2.5 (95\% $\mathrm{Cl}$ 1.2-5.1) versus the inpatient reference individuals, and an HR of 3.9 (95\% Cl 2.2-7.0), versus the general population, respectively. In the same study it was proposed that CD subjects are at risk of sepsis from other bacterial infections than pneumococcal disease and suggested that other mechanisms apart from hyposplenism may contribute to increase this risk. Walters et al [26], in their commentary about data from United Kingdom and Swedish Celiac Disease registers, found that the risk of sepsis in these patients was higher than hip fracture and lymphoma. Peters et al. [27], in a Swedish study on 10,032 Swedish celiac patients hospitalized from January 1964 to December 1993, reported 818 celiac patient deaths, with a Standardized Mortality Ratio (SMR) for pneumonia of 2.9.

Grainge et al. [28], in a prospective cohort study, assessed an approximately $37 \%$ higher risk of death in CD patients than in the general population. The study, performed in southern Derbyshire (United Kingdom) included 1,092 celiac patients. A statistically significant increase in all-cause mortality was observed (SMR 1.37, 95\% Cl 1.16-1.62), and respiratory disease played an important role (SMR 1.57, 21 deaths, 11 due to pneumonia). More than half of the deaths from respiratory diseases were thus caused by pneumonia, suggesting that vaccination may be a possible strategy to reduce mortality resulting from pneumococcal infection.

These conclusions were not confirmed in another study, by Abdul Sultan et al. [29]; these Authors performed a similar study on the British population, quantifying the excess cause-specific mortality between the CD population and the general population, with a follow-up of 10 years after CD diagnosis. Their analysis did not show the excess of mortality risk indicated by other studies in the literature (as in the case of cancer, cardiovascular disease, etc.) and their conclusions were also valid for respiratory diseases. The authors concluded that the results should reassure patients and practitioners that there is no risk of excess cause-specific mortality in CD patients compared to the general population, except for a slight excess risk of death from non-Hodgkin's lymphoma.

More recently, Zingone et al. [30] evaluated the risk of community-acquired pneumonia in CD patients; they studied 9,803 patients with celiac disease and 101,755 controls and found 179 and 1,864 community-acquired pneumonia events, respectively. The absolute rate of pneumonia was similar in both celiac disease patients and controls: 3.42 and 3.12 per 1,000 person-years, respectively (HR 1.07, 95\% Cl 0.91-1.24). However, there was a $28 \%$ increased risk of pneumonia in unvaccinated celiac disease subjects compared to unvaccinated controls (HR 1.28, 95\% Cl 1.02-1.60). On the other hand, $C D$ patients vaccinated against pneumococcal infection did not present this risk ( $\mathrm{HR} 0.88,95 \% \mathrm{Cl} 0.70-1.10)$. The authors concluded that the unvaccinated subjects were exposed to a greater risk and that vaccination should be a safe and effective strategy to limit this risk.

Emilsson et al. [31] assessed whether intestinal mucosal healing or persistent villous atrophy could influence the risk of infections in CD patients. The authors found no significant differences between the two groups regarding any type of infection, and intestinal mucosal healing did not influence the risk of serious infections requiring hospital-based medical treatment. The authors did not evaluate hyposplenism and suggested that hyposplenism may persist even after intestinal mucosal healing. This could explain why there is no difference in the risk of infection between the two groups.

Finally, a recent meta-analysis [10] evaluated the risk of pneumococcal infection compared to the general population, showing a higher risk in $C D$ patients than in the general population (Odds Ratio, OR, 1.66, 95\% Cl 1.43-1.92).

\section{International experience of pneumococcal vaccination}

Data on vaccination in CD patients are only available from England. A retrospective audit [32] showed that only $19.3 \%$ of celiac patients aged below 65 had received the pneumococcal vaccine in 2013, and these data were confirmed by Zingone et al. [30] who reported that only $26.6 \%$ of CD patients had received vaccination after the diagnosis.

Better vaccination rates were demonstrated by Di Sabatino et al. [33] in a different setting; they registered vaccination coverage, mortality and infection rates in all patients who underwent splenectomy over a 6-year period (2004-2009). In this study, reasons for splenectomy in the 216 identified patients were, in order: hematologic disorders, solid tumors, traumatic rupture, and other causes. Only $67.6 \%$ patients received at least one of the four vaccines (against S. pneumoniae, N. meningitidis, $H$. influenzae type $B$, and influenza). Obviously, this indicated a poor compliance with recommended vaccination. Overall, the 
mortality rate was significantly greater in unvaccinated than in vaccinated patients. Of the 21 reported cases of overwhelming post-splenectomy infection, 8 were fatal and 5 potentially vaccine-preventable.

\section{Type of vaccine}

Many types of vaccines are available on the market and it is currently unclear which type of vaccination is preferable in $C D$ patients. Some scientific and health authorities recommend the use of PCV-10 and PCV-13 (pneumococcal conjugate vaccine, valent-10 and -13) in children and adult patients with alterations of innate and adaptive immunity. Furthermore, the CRM197 diphtheria protein (i.e. the carrier protein for the conjugate vaccine) changes the nature of the response from T-independent to T-dependent, making this vaccine particularly suitable in infants, especially below the age of 2 years, when the splenic IgM-memory B cell pool is still immature. Similarly, adult hyposplenic patients, in whom the IgMmemory B cell pool is depleted, would benefit from PCV-13, as its T-dependent mechanism bypasses the immunological impairment due to the lack of IgM-memory B cells [34]. However, this kind of vaccine is recommended by current guidelines only in infancy. PPV 23 (pneumococcal capsular polysaccharide vaccine), whose protective action is based on the production of opsonizing anti-capsular antibodies by means of a T-independent mechanism, is at present recommended in asplenic/hyposplenic children above 5 years old and in adults, since it is associated with a poor or absent response in children under 5 years $[35,36]$.

On the other hand, the current international literature also suggests using pneumococcal conjugate vaccine instead of the capsular polysaccharide vaccine in adult patients with hyposplenia or asplenia due to its T cell-dependent mechanism, which should be not compromised in these clinical conditions [21]. Therefore, in naïve patients PCV-13 (1 dose) is recommended, followed by PPV23 (1 dose) at least 8 weeks later. In patients who have previously received PPV-23, PCV-13 is administered $\geq 1$ year later. A booster dose of PPV-23 after 5 years is also suggested [37].

\section{Do we vaccinate all cd patients?}

To date, no subgroup of subjects at greater risk of pneumococcal infection has been identified within the group of $C D$ patients. A limitation bias of previous studies is the lack of analysis of splenic function and, more generally, of the quantification of the immunological deficit in CD patients, which can predispose to an increased risk of invasive pneumococcal disease. However, an analysis of splenic function and size is not routinely performed in CD patients [11]. It is important to underline that asplenia is a risk factor strongly associated with invasive pneumococcal disease, quantified by Backhaus et al. [38] in a recent study as $\mathrm{RR}=14.08(95 \% \mathrm{Cl} 10.38-19.10)$.

We can suggest two practical approaches to select CD patients who could benefit from vaccination, thus avoiding the necessity to vaccinate all newly diagnosed $C D$ patients and consequently saving money. First, from a simple, clinical point of view, it is reasonable to vaccinate $C D$ patients with advancing age at diagnosis, concomitant AID, CD complications (refractory
$C D$, ulcerative jejuno-ileitis, collagenous sprue), previous history of major infections/sepsis and/or thromboembolism, and splenic atrophy [11]. Second, the presence of a small-sized spleen could be investigated by abdominal ultrasound, which can be included in the routine evaluation of patients with suspected CD. Evaluating the percentage of pitted erythrocyte levels in blood films can be a further way to help decide whether or not to vaccinate patients.

\section{Conclusion}

At present, there is no study in the literature on $C D$ patients with hyposplenism that quantifies the risk of invasive pneumococcal disease, even if the functional impairment of the spleen is a wellknown risk factor for pneumococcal infection [21].

Most authors, in accordance with the British Society of Gastroenterology guidelines, suggest that pneumococcal vaccination in CD patients is a safe and effective method for the prevention of pneumococcal infections [20]. However, studies in the literature show conflicting data on the excess risk of pneumonia, invasive pneumococcal disease, sepsis and mortality in CD patients compared to the general population.

In the 14-65 age group there is in fact an excess of risk, in part likely due to a lack of pneumococcal vaccination, in the absence of vaccination schemes [10].

It is necessary to identify those subgroups of CD patients that could be exposed to a greater risk on the basis of the course of $C D$ and degree of splenic function deficiency. A systematic evaluation of spleen function would be useful in $C D$ to select the patients who may benefit from vaccination, but it is not currently performed and there are no unanimous indications about the methods to be used.

At present, in the international literature, there are no studies to verify whether gluten and active CD can affect the pneumococcal vaccination. Anania et al. [39] showed that immunological responses to other vaccines in $C D$ children are not different from those in the general population, except those for hepatitis B.

In conclusion, pneumococcal vaccination should be performed in $C D$ patients, considering the increased risk of invasive pneumococcal disease in unvaccinated CD patients, as well as the safety and efficacy profile of the PCV vaccine in asplenic subjects. However, it does not seem logical, nor economically viable, to vaccinate all newly diagnosed $C D$ patients. Other strategies to identify, and thus to protect, CD patients at risk should be evaluated and applied on a large scale.

\section{Expert opinion}

What are the key weaknesses in clinical management so far?

Currently, hyposplenism, a well-known risk factor for pneumococcal infection, is not routinely researched and evaluated in celiac patients.

\section{What potential does further research hold? What is the ultimate goal in this field?}

A systematic evaluation of spleen function should be useful in $C D$ to select the patients who may benefit from vaccination, to reduce the risk of invasive pneumococcal disease. 


\section{What research or knowledge is needed to achieve this goal and what is the biggest challenge in this goal being achieved?}

Considering that hyposplenism affects more than one-third of celiac patients, and that it is strongly related to a higher risk of infections, every physician should investigate for signs of hyposplenism on peripheral blood smear in CD patients. The finding of an atrophic spleen on abdominal ultrasound should then lead the physician to assess spleen function.

\section{Is there any particular area of the research you are finding of interest at present?}

It might be of practical interest to better explore the mechanism by which malnutrition and increased intestinal permeability may predispose to an impairment of the immune system's ability to respond to infections in celiac patients, and how a gluten-free diet reverses immunological impairment in celiac patients.

\subsection{Five-year view}

It is reasonable to speculate that within the next five years hyposplenism will be routinely researched in celiac patients and that all celiac patients with impaired immunologic function will undergo a complete vaccination protocol, including S. pneumoniae, N. meningitidis, H. influenzae type $B$, and influenza.

\section{Funding}

This paper was not funded.

\section{Declaration of interest}

The authors have no relevant affiliations or financial involvement with any organization or entity with a financial interest in or financial conflict with the subject matter or materials discussed in the manuscript. This includes employment, consultancies, honoraria, stock ownership or options, expert testimony, grants or patents received or pending, or royalties.

\section{Reviewer disclosures}

Peer reviewers on this manuscript have no relevant financial or other relationships to disclose

\section{References}

Papers of special note have been highlighted as either of interest $(\cdot)$ or of considerable interest $(\cdot \bullet)$ to readers.

1. Lebwohl B, Sanders DS, Green PHR. Celiac disease. Lancet. 2018;391:70-81.

2. Kneepkens CM, von Blomberg BM. Clinical practice: celiac disease. Eur J Pediatr. 2012;171:1011-1021.

3. Saalman R, Fällström SP. High incidence of urinary tract infection in patients with celiac disease. Arch Dis Child. 1996;74:170-171.

4. Ludvigsson JF, Sanders DS, Maeurer M, et al. Risk of tuberculosis in a large sample of patients with celiac disease -a nationwide cohort study. Aliment Pharmacol Ther. 2011;33:689-696.

5. Thomas $\mathrm{HJ}$, Wotton $\mathrm{CJ}$, Yeates $\mathrm{D}$, et al. Pneumococcal infection in patients with celiac disease. Eur J Gastroenterol Hepatol. 2008:20:624-628.

6. Röckert Tjernberg A, Bonnedahl J, Inghammar M, et al. Celiac disease and invasive pneumococcal disease: a population-based cohort study. Epidemiol Infect. 2017;145:1203-1209.
7. Ludvigsson JF, Olén $\mathrm{O}$, Bell $\mathrm{M}$, et al. Celiac disease and risk of sepsis. Gut. 2008;57:1074-1080.

8. Bullen AW, Hall R, Gowland G, et al. Hyposplenism, adult celiac disease, and autoimmunity. Gut. 1981;22:28-33.

9. Harms Pritchard G, Pepper M. Memory B cell heterogeneity: remembrance of things past. J Leukoc Biol. 2018;103:269-274.

10. Simons M, Scott-Sheldon LAJ, Risech-Neyman Y, et al. Celiac disease and increased risk of pneumococcal infection: a systematic review and meta-analysis. Am J Med. 2018;131:83-89.

.- Recent and comprehensive review on CD and Pneumococcal infection.

11. Di Sabatino A, Brunetti L, Carnevale MG, et al. Is it worth investigating splenic function in patients with celiac disease? World J Gastroenterol. 2013;19:2313-2318.

12. Di Sabatino A, Rosado MM, Ciccocioppo R, et al. Depletion of immunoglobulin $M$ memory $B$ cells is associated with splenic hypofunction in inflammatory bowel disease. Am J Gastroenterol. 2005;100:1788-1795.

13. Di Sabatino A, Carnevale Maffè G, Brunetti L, et al. Splenic hypofunction in patients with an incidental finding of small-sized spleen at abdominal ultrasound. Intern Emerg Med. 2013;8:361-362.

- A simple "Letter to the Editor" which suggests a practical approach to the problem.

14. Corazza GR, Zoli G, Di Sabatino A, et al. A reassessment of splenic hypofunction in celiac disease. Am J Gastroenterol. 1999;94:391-397.

15. Di Sabatino A, Rosado MM, Cazzola P, et al. Splenic hypofunction and the spectrum of autoimmune and malignant complications in celiac disease. Clin Gastroenterol Hepatol. 2006:4:179-186.

16. Bjarnason I, Marsh MN, Price A, et al. Intestinal permeability in patients with celiac disease and dermatitis herpetiformis. Gut. 1985;26:1214-1219.

17. Wierdsma NJ, van Bokhorst-de van der Schueren MA, Berkenpas $M$, et al. Vitamin and mineral deficiencies are highly prevalent in newly diagnosed celiac disease patients. Nutrients. 2013;5:3975-3992.

18. Doig CJ, Sutherland LR, Sandham JD, et al. Increased intestinal permeability is associated with the development of multiple organ dysfunction syndrome in critically ill ICU patients. Am J Respir Crit Care Med. 1998;158:444-451.

19. Robertson DA, Taylor N, Sidhu H, et al. Pulmonary permeability in celiac disease and inflammatory bowel disease. Digestion. 1989;42:98-103.

20. Ludvigsson JF, Bai JC, Biagi F, et al. BSG celiac disease guidelines development group; British society of gastroenterology. Diagnosis and management of adult celiac disease: guidelines from the British society of gastroenterology. Gut. 2014;63:1210-1228.

- One of the most recent and comprehensive guidelines on Celiac Disease.

21. William BM, Corazza GR. Hyposplenism: a comprehensive review. Part I: basic concepts and causes. Hematology. 2007;12:1-13.

22. Rubio-Tapia A, Hill ID, Kelly CP, et al. American college of gastroenterology. ACG clinical guidelines: diagnosis and management of celiac disease. Am J Gastroenterol. 2013;108:656-676.

23. O'Donoghue DJ. Fatal pneumococcal septicaemia in celiac disease. Postgrad Med J. 1986;62:229-230.

24. Parnell N, Thomas P. Fatal pneumococcal septicaemia in a celiac patient. Eur J Gastroenterol Hepatol. 1998;10:899-900.

25. Ouseph MM, Simons M, Treaba DO, et al. Fatal streptococcus pneumoniae sepsis in a patient with celiac disease-associated hyposplenism. ACG Case Rep J. 2016;3:e140.

26. Walters JR, Bamford KB, Ghosh S. Celiac disease and the risk of infections. Gut. 2008;57:1034-1035.

27. Peters U, Askling J, Dridley G, et al. Causes of death in patients with Celiac disease in a population-based Swedish cohort. Arch Intern Med. 2003;163:1566-1572.

28. Grainge MJ, West J, Card TR, et al. Causes of death in people with celiac disease spanning the pre- and post-serology era: a 
population-based cohort study from Derby, UK. Am J Gastroenterol. 2011;106:933-939.

29. Abdul Sultan A, Crooks CJ, Card T, et al. Causes of death in people with celiac disease in England compared with the general population: a competing risk analysis. Gut. 2015;64:1220-1226.

30. Zingone F, Abdul Sultan A, Crooks CJ, et al. The risk of community-acquired pneumonia among 9803 patients with celiac disease compared to the general population: a cohort study. Aliment Pharmacol Ther. 2016;44:57-67.

31. Emilsson L, Lebwohl B, Green PH, et al. Mucosal healing and the risk of serious infections in patients with celiac disease. United European Gastroenterol J. 2018;6:55-62.

32. Khan J, Jennings A, Subramanian S. OC-021 A retrospective audit of pneumococcal \& influenza vaccination in celiac disease. Gut. 2013;62:A9-A10.

33. Di Sabatino A, Lenti MV, Tinozzi FP, et al. Vaccination coverage and mortality after splenectomy: results from an Italian single-centre study. Intern Emerg Med. 2017;12:1139-1147.

34. Durando P, Alicino C, De Florentiis D, et al. Improving the protection against Streptococcus pneumoniae with the new generation 13-valent pneumococcal conjugate vaccine. J Prev Med Hyg. 2012;53:68-77.

35. World Health Organization. Pneumococcal vaccines WHO position paper-2012. Wkly Epidemiol Rec. 2012;87:129-144.

36. American Academy of Pediatrics Committee on Infectious Diseases. Recommendations for the prevention of Streptococcus pneumoniae infections in infants and children: use of 13-valent pneumococcal conjugate vaccine (PCV13) and pneumococcal polysaccharide vaccine (PPSV23). Pediatrics. 2010;126:186-190.

37. Bonanni P, Chiamenti G, Conforti G, et al. Scientific board of "lifetime immunization schedule". The 2016 lifetime immunization schedule, approved by the Italian scientific societies: A new paradigm to promote vaccination at all ages. Hum Vaccin Immunother. 2017;13:2531-2537.

38. Backhaus E, Berg S, Andersson R, et al. Epidemiology of invasive pneumococcal infections: manifestations, incidence and case fatality rate correlated to age, gender and risk factors. BMC Infect Dis. 2016;16:367.

- A comprehensive review of Pneumococcal infections.

39. Anania C, Olivero F, Spagnolo A, et al. Immune response to vaccines in children with celiac disease. World J Gastroenterol. 2017;23:3205-3213. 